\title{
Investigar para institucionalizar el aprendizaje servicio en la universidad española
}

\author{
María del Mar Lorenzo Moledo, Ígor Mella Núñez, Jesús García Álvarez, \\ Cristina Varela Portela \\ Universidad de Santiago de Compostela, España
}

\section{Resumen}

La investigación en aprendizaje servicio en España se ha caracterizado por la ausencia de estudios que empleen diseños experimentales, por carecer de datos longitudinales de los efectos a largo plazo, por utilizar muestras pequeñas y porque buena parte de los estudios se basan en las percepciones de alumnos y profesores. Justamente, partiendo de esta realidad, nuestro objetivo en este trabajo es presentar el diseño de una investigación experimental centrada en estudiar la pertinencia del aprendizaje servicio como marco de innovación y desarrollo de los procesos de aprendizaje en las universidades españolas y evaluar la eficacia del aprendizaje servicio para mejorar las competencias sociales y las destrezas cívicas así como los conocimientos técnicos (contenidos disciplinares) de los estudiantes universitarios.

\section{Palabras clave}

Aprendizaje servicio, institucionalización, universidad, investigación.

Fecha de recepción: 9/II/2017

Fecha de aceptación: 9/II/2017 


\title{
Research aimed at institutionalizing service learning in the Spanish university system
}

\begin{abstract}
Service learning research in Spain has been characterized by the absence of studies using experimental designs due to a lack of longitudinal data regarding long-term effects, to the use of small samples and because many of these studies are based on students and teachers' perceptions. Given this context, the aim of this work is to present the design of an experimental research focused on studying the relevance of service learning as a framework for innovation and development of learning processes in Spanish universities; and also to evaluate the effectiveness of service learning in order to improve social competences and civic skills, as well as technical knowledge (disciplinary content) of university students.
\end{abstract}

\section{Keywords}

Service learning, institutionalization, university, research. 


\section{Introducción}

Distintas instancias internacionales afirman la necesidad de que la universidad cree las condiciones adecuadas para fomentar un aprendizaje más centrado en el estudiante y haga uso de métodos de enseñanza innovadores. Así lo reconocen los Ministros de Educación de los países implicados en la configuración del Espacio Europeo de Educación Superior (EEES), en la Declaración de Bucarest (2012).

Ciertamente, uno de los métodos que más se focaliza en torno al aprendizaje del estudiante es el aprendizaje servicio (ApS), donde los alumnos aprenden mientras actúan sobre necesidades reales del entorno con la finalidad de mejorarlo. En una propuesta de aprendizaje servicio se combinan, pues, el aprendizaje de contenidos académicos con el entrenamiento en la disponibilidad (habilidades) para movilizarlos en contextos reales (Puig, 2009; Santos Rego, Sotelino, y Lorenzo, 2015). El alumnado tiene así la oportunidad de transferir esos aprendizajes para atender necesidades concretas.

Es justo reconocer la existencia de un extenso acuerdo a la hora de considerar la vertiente académica como la principal finalidad de las instituciones de educación superior. No obstante, en una sociedad del aprendizaje y una economía del conocimiento como las actuales, se le exige a la universidad que ofrezca una formación integral que redunde no solo en una formación técnica de calidad sino también en un aumento de la responsabilidad social y el compromiso cívico del alumnado (McIlrath, Lyons, y Munck, 2012; Santos Rego y Lorenzo, 2007). Se trata, pues, de formar a los estudiantes universitarios no solo como profesionales sino también como ciudadanos, exigencia demandada por el EEES y que reclama romper con los patrones clásicos del sistema universitario (Martínez, 2008).

Así pues, el objetivo de este trabajo no es otro que presentar el diseño y desarrollo de una investigación centrada en estudiar la pertinencia del aprendizaje servicio como marco de innovación y desarrollo de los procesos de aprendizaje en las universidades españolas; y evaluar la eficacia del aprendizaje servicio para mejorar las competencias sociales y las destrezas cívicas así como los conocimientos técnicos de los estudiantes universitarios ${ }^{1}$.

Lo que pretendemos es validar un modelo de institucionalización de la metodología del aprendizaje servicio en la universidad española. En nuestro contexto, a pesar del incremento de iniciativas que trabajan en pro de la institucionalización de esta metodología, el discurrir del ApS ha sido lento, a diferencia de lo acontecido en otras latitudes, caso de los Estados Unidos de América, donde la institucionalización es no solo palpable, sino objeto de preocupación y estudio consistente (Bringle y Hatcher, 2000; Furco, 2007). Además, para este modelo de institucionalización que

\footnotetext{
${ }^{1}$ www.usc.es/apsuni
} 
defendemos es necesario, cuando menos, trabajar a dos niveles: el ApS como filosofía, y el ApS como programa (Puig, Palos, Martín, Rubio, y Escofet, 2016).

Ahora bien, no son pocos los trabajos que se han publicado y que, en sentido estricto, carecen de evidencias empíricas consistentes al respecto, basando buena parte de sus afirmaciones, y aún conclusiones, en las experiencias (por supuesto, valiosas en la mayoría de los casos), de profesores bien dispuestos al uso de la mencionada metodología. Y es así como llegan a explicar buenos resultados en sus alumnos, sin que medie un diseño riguroso de investigación.

Sin embargo, si lo que pretendemos es afirmar las posibilidades del aprendizaje-servicio en el contexto universitario español, lo primero es no olvidar que una dinámica de instalación como la que se pretende exige un grado suficiente de conocimiento sobre aquellos elementos y factores que podrán determinar el éxito en la introducción de esta metodología. Para ello, en este trabajo se presenta el proceso de institucionalización del ApS seguido en la Universidad de Santiago de Compostela, articulado en torno a un proyecto de investigación. Se expone el procedimiento por el cual se diseñó y se está implementando y evaluando (con instrumentos cualitativos y cualitativos) un programa que incluye a 20 experiencias de aprendizaje-servicio.

\section{EI ApS en la educación superior: la institucionalización como desafío}

Para comenzar, podemos definir el ApS como aquella acción educativa en la que los estudiantes, previa identificación de una necesidad, realizan algún tipo de servicio, gracias al cual mejoran su conocimiento de una disciplina y amplían su sentido de responsabilidad social (Ugarte y Naval, 2010). Esta definición nos sitúa en posición de afirmar que los programas de aprendizaje servicio se asocian, en su definición, aplicación y desarrollo, a patrones de mejora cognitiva y social del alumnado. Así, la propia denominación de esta metodología da buena cuenta de su dualidad epistémica: por un lado, aprendizaje en tanto que dimensión asociada al rendimiento académico de los estudiantes; y, por otro, servicio como dimensión vinculada al compromiso cívico del alumnado (Santos Rego et al., 2015).

Esta dualidad se ve reflejada en la bibliografía al respecto. Un primer ejemplo es el de Tapia (2004), cuando afirma que el ApS supone, además de un servicio de calidad a la comunidad, la firme integración del aprendizaje disciplinar en dicho servicio. Del mismo modo, Howard (2003) identifica tres elementos esenciales en el aprendizaje servicio: la provisión a la comunidad de un servicio que da respuesta a una necesidad que fue identificada en su seno, el fortalecimiento del aprendizaje académico del alumnado $y$, en último lugar, el incremento de la responsabilidad social y ciudadana además de la participación activa de los estudiantes en su comunidad. Pero esta dicotomía que caracteriza al impacto del ApS en el alumnado no se establece de 
un modo disociado, sino que, siguiendo el paradigma establecido por Furco (2005), en realidad el vínculo existente entre el aprendizaje servicio y el rendimiento académico se establece como consecuencia de los efectos que esta práctica tiene en una serie de factores mediadores: autoestima, empoderamiento, conducta prosocial, motivación y compromiso.

Además, el efecto y los beneficios del aprendizaje servicio pueden ir más allá de los estudiantes y de los potenciales resultados cognitivo-sociales en los mismos, ya que es posible que alcancen al profesorado, a la comunidad y a la universidad en su conjunto, máxime si consigue vincular tres de sus inequívocas misiones: docencia, investigación y responsabilidad social. De sus teóricas ventajas se ha hecho eco en nuestro país la Conferencia de Rectores de Universidades Españolas (CRUE) con la aprobación de un documento técnico sobre institucionalización del aprendizaje servicio como estrategia docente dentro del marco de la responsabilidad social universitaria para la promoción de la sostenibilidad en la universidad.

Sin embargo, toda práctica que se deriva de la universidad, especialmente aquellas que como el ApS suponen una transformación pedagógica, requieren la incorporación de recursos, ayudas y estrategias que den soporte a la introducción de nuevas actividades y metodologías. Podemos diferenciar así dos factores que se han de implicar necesariamente en el proceso de institucionalización del aprendizaje servicio en la universidad: por un lado, la integración de la participación en experiencias de ApS en su tejido (por ejemplo como un requisito para que los estudiantes se gradúen); y por otro, su formalización a través de la creación de estructuras organizacionales y medidas de rendición de cuentas (Stater y Fotheringham, 2009).

En la misma línea, Bringle y Hatcher (2000) demostraron en su estudio que en el proceso de institucionalización del aprendizaje servicio es conveniente considerar ciertos aspectos que determinarán el éxito del proceso. El primero de ellos es el respaldo de agencias o iniciativas (en su caso Campus Compact) que asesoran a las instituciones de educación superior a la hora de enfrentar este proceso de cambio paradigmático desde un punto de vista educativo. Destacan también la importancia que tiene desarrollar infraestructuras en las universidades para dar soporte a las actividades de aprendizaje servicio, como puede ser una oficina centralizada que ofrezca apoyo técnico y logístico, incentivos económicos y reconocimiento.

Por tanto, la institucionalización del aprendizaje servicio no es una tarea a emprender de modo aislado y puntual, sino que es un proceso laborioso y constante en el que se han de superar fases consecutivas aunque interdependientes. Así lo recoge Furco (2011) al reconocer tres etapas en el desarrollo de este proceso (Tabla 1 ).

\begin{tabular}{|l|l|}
\hline \multicolumn{1}{|c|}{ Etapa } & \multicolumn{1}{c|}{ Nivel de desarrollo } \\
\hline $\begin{array}{l}\text { 1. Creación de } \\
\text { masa crítica }\end{array}$ & $\begin{array}{l}\text { Las instituciones } \\
\text { universitarias comienzan a } \\
\text { reconocer el ApS y a } \\
\text { construir las bases de las }\end{array}$ \\
\hline
\end{tabular}




\begin{tabular}{|l|l|}
\hline & $\begin{array}{l}\text { estructuras de apoyo en } \\
\text { toda la universidad. }\end{array}$ \\
\hline $\begin{array}{l}\text { 2. Construcción } \\
\text { de calidad }\end{array}$ & $\begin{array}{l}\text { Las universidades buscan } \\
\text { asegurar el desarrollo de } \\
\text { experiencias de ApS de } \\
\text { calidad, sobreponiendo } \\
\text { esta última a la cantidad } \\
\text { de proyectos. }\end{array}$ \\
\hline $\begin{array}{l}\text { 3. Sustentabilidad } \\
\text { institucional }\end{array}$ & $\begin{array}{l}\text { La universidad tiene ya } \\
\text { completamente } \\
\text { institucionalizado el ApS. }\end{array}$ \\
\hline
\end{tabular}

Tabla 1. Etapas de desarrollo en la institucionalización del ApS. Fuente: elaboración propia a partir de Furco (2011)

En definitiva, la bibliografía existente al respecto nos sirve de soporte para formular la principal hipótesis del proyecto de investigación presentado en este trabajo: la institucionalización del aprendizaje servicio en la universidad española contribuirá a los procesos de innovación docente, a la mejora de la calidad y al aprendizaje de competencias académicas y sociales que favorecen la inserción laboral y el desarrollo de una ciudadanía más activa.

\section{La investigación como medio para la institucionalización del ApS}

En primer lugar, es preciso remarcar que el proyecto de investigación que presentamos supone una fase, ciertamente avanzada, dentro de un proceso de institucionalización más amplio. En realidad, en la USC este camino se inició dando a conocer el ApS a la comunidad universitaria a través de múltiples acciones. Esta primera fase de difusión se configuró a través de la organización por parte del Grupo de Investigación ESCULCA de una serie de actividades de carácter formativo, que llegaron a todos los niveles educativos
(Santos Rego et al., 2015). Entre dichas iniciativas destacamos los cursos de formación de profesorado universitario sobre la metodología de ApS (incluidos en el Plan de Formación e Innovación Docente de la USC desde el curso 2011-2012); además del seminario Aprendizaje servicio en la enseñanza no universitaria. El valor de la experiencia (celebrado desde 2015 con periodicidad anual).

Este proceso de difusión se consolidó en el año 2015, cuando la Red de Excelencia Universidad, Innovación y Aprendizaje en la Sociedad del Conocimiento, formada por 4 universidades y coordinada desde la USC, organizó el I Simposio Internacional de aprendizaje servicio en la educación superior: innovación, calidad e institucionalización (2015) destinado al profesorado de las tres universidades del sistema universitario de Galicia. Este dinamismo formativo emprendido por la Universidad de Santiago de Compostela dio lugar, asimismo, a la aparición de las primeras experiencias de aprendizaje servicio que se desarrollaron en esta universidad y culminó, en el año 2016, con la organización del VII Congreso Nacional y II Internacional de Aprendizaje Servicio Universitario. Del mismo modo, en este período se han realizado numerosas publicaciones científicas sobre el tema, además de la defensa de dos tesis doctorales centradas en analizar la situación del ApS en la USC.

Por tanto, podemos entender el proyecto de investigación que aquí abordamos como una fase avanzada de 
un proceso más amplio, con el que se pretende dotar de evidencias a un proceso de institucionalización que no puede, o por lo menos no debe, ser el resultado de meras convicciones y buenas intenciones sin respaldo empírico. Por ello, y teniendo en cuenta los objetivos anteriormente enunciados, la investigación se plantea bajo parámetros metodológicos diversos (Metodología Combinada Integradora Exploratoria) y con una muestra de centros de seis universidades españolas. El estudio se desarrolla en tres fases interdependientes, pero metodológicamente diferenciadas.

En la primera fase se realizó un estudio exploratorio y descriptivo, con la intención de analizar la presencia del aprendizaje servicio como filosofía en la universidad. Se emplearon una serie de instrumentos y técnicas expresamente diseñados para abordar esta tarea en las seis universidades (Buenestado, Álvarez, y González, 2016; Pérez, Vázquez, y López, 2016; Santos Rego, Jover, Naval, Alvarez, Vázquez, y Sotelino, en prensa) (Tabla 2).

\begin{tabular}{|l|l|}
\hline \multicolumn{1}{|c|}{ Instrumentos } & \multicolumn{1}{c|}{ Muestra } \\
\hline $\begin{array}{l}\text { Análisis de contenido de } \\
\text { planes estratégicos de } \\
\text { formación del profesorado } \\
\text { en las universidades }\end{array}$ & $\begin{array}{l}\text { 6 programas } \\
\text { institucionales de } \\
\text { formación del } \\
\text { profesorado } \\
\text { universitario }\end{array}$ \\
\hline $\begin{array}{l}\text { Análisis de contenido de } \\
\text { programas/guías docentes } \\
\text { de las materias }\end{array}$ & $\begin{array}{l}174 \text { programas de } \\
\text { materias de grado }\end{array}$ \\
\hline $\begin{array}{l}\text { Cuestionario para analizar } \\
\text { la cultura docente en la } \\
\text { universidad }\end{array}$ & $\begin{array}{l}1916 \text { profesores } \\
\text { universitarios }\end{array}$ \\
\hline Entrevista a decanos & $\begin{array}{l}42 \text { decanos de } \\
\text { facultades }\end{array}$ \\
\hline
\end{tabular}

Tabla 2. Instrumentos y muestra del estudio exploratorio. Fuente: elaboración propia.
Esta primera fase se vio complementada con la realización de dos grupos de discusión con empleadores de graduados universitarios. Se trataba de reconocer la vinculación existente entre el desarrollo de competencias cívicosociales en la universidad y la inserción profesional de los estudiantes.

La segunda fase del proyecto se centra en el diseño del programa de ApS para la universidad, siguiendo la siguiente secuencia:

1. Diagnóstico-detección. Hemos detectado las experiencias de ApS que se están llevando a cabo en las seis universidades, pero también aquellas que sin ser ApS podrían asemejarse, e incluso al profesorado dispuesto a iniciarse en esta metodología. También pretendimos identificar qué características definen una experiencia de ApS como de alta calidad. Además de los datos obtenidos gracias a los instrumentos de la primera fase, se movilizaron otros recursos que nos permitieron acceder a las experiencias ya en desarrollo:

- La información facilitada por las oficinas/unidades de formación docente de las universidades, además del registro de los profesores participantes a cursos sobre ApS.

- La revisión de la asistencia y participación de docentes de estas universidades en los congresos sobre ApS universitario en España, además 
de la pertenencia a la Red Universitaria de Aprendizaje Servicio.

- Por último, el conocimiento directo de alguna de las experiencias.

Así, se diseñó una Ficha de Registro de Experiencias de ApS y una rúbrica para proceder al análisis de los datos obtenidos. Se consiguieron finalmente un total de 42 respuestas de las seis universidades.

2. Diseñar un programa y movilizar recursos que faciliten y hagan sostenible su implementación. Para ello se está trabajando en varias líneas:

- Plan de Formación-Innovación Docente. Se trata de un plan con tres núcleos: la formación básica sobre ApS y los elementos básicos para diseñar un proyecto (6 horas); el diseño por parte de los docentes de su proyecto de ApS (4 horas); y, por último, el desarrollo tutorizado de cada una de las experiencias, y su evaluación.

- Reconocimiento institucional. El plan de formación y el trabajo de los profesores debe ser reconocido institucionalmente. De hecho, varias universidades del proyecto han puesto en marcha distintas acciones orientadas a este fin. En el caso de la USC se ha aprobado la I Convocatoria de proyectos de innovación educativa en aprendizaje-servicio (ApS).

- Partenariado y comunidad educativa. Se debe facilitar a los docentes la búsqueda de socios comunitarios con los que trabajar. Esta tarea se ve apoyada por el Servicio de Participación e Integración Universitaria (SEPIU) de la USC y su red de entidades colaboradoras.

- La universidad debe tener una estructura organizativa que permita visibilizar el ApS, favorecer la coordinación de entidades, gestión de convenios. Esta infraestructura se ve configurada por el ya nombrado SEPIU y, especialmente, por el Centro de Tecnologías para el Aprendizaje (CeTA) ${ }^{2}$.

El plan de formación se ha desarrollado en la USC, concluyendo con un total de 25 proyectos diseñados. Cada proyecto ha sido sometido a una evaluación del diseño, implicando a expertos en ApS (criterio interjueces).

La tercera fase supone la implementación y evaluación, según el diseño establecido, de cada uno de los proyectos de ApS en la Universidad de Santiago de Compostela a modo de estudio piloto, con el fin de validar un modelo transferible a las otras cinco universidades participantes, así como al conjunto del sistema universitario español. Finalmente, se están

\footnotetext{
${ }^{2}$ http://www.usc.es/es/servizos/ceta/innovacion/ aps/index.html
} 
implementando 20 proyectos de ApS en el curso 2016-2017: 1 en el área de Humanidades, 2 en Técnicas, 3 en Ciencias de la Salud, y 14 en Ciencias Sociales y Jurídicas.

Por lo que se refiere a la evaluación de estos 20 proyectos, se ha seguido un diseño cuasi-experimental de dos grupos no equivalentes con pretest y postest y la participación en los proyectos como variable independiente. El poder comparar a estos dos grupos de estudiantes es uno de los potenciales del proyecto presentado, ya que ciertos estudios informan de los beneficios derivados de la implementación de prácticas de ApS en la universidad, empleando como principal criterio de evaluación la comparación entre alumnos que participan en estas prácticas y alumnos que no lo hacen (Astin, Vogelgesang, Ikeda y Yee, 2000; Gallini y Moely, 2003). No obstante, la existencia de este tipo de investigaciones se ha constatado principalmente en países anglosajones, y no así en España.

Se han diseñado instrumentos para evaluación inicial, de implementación y final, con la intención de analizar el efecto de las experiencias en alumnos, profesores, universidad y comunidad.

Se trata de instrumentos cuantitativos y cualitativos, ya que diversos autores plantean la necesidad de que el enfoque cuantitativo sea complementado con un punto de vista cualitativo que permita captar las características propias de cada experiencia individual de aprendizaje-servicio, pudiendo así confirmar o refutar los datos cuantificables (Furco, 2003; Howard,
2003). De este modo, se diseñaron los siguientes instrumentos:

- Evaluación inicial:

- Cuestionario sobre competencias cívico-sociales y autoeficacia del alumnado universitario (CUCOCSA).

- Entrevista inicial con el profesorado responsable del proyecto de ApS.

- Evaluación de la implementación:

- Portafolio de los estudiantes.

- Entrevista de seguimiento con el profesorado responsable.

- Evaluación de los resultados:

- Diferencial semántico para medir la satisfacción del alumnado.

- Entrevista grupal con el alumnado participante en cada proyecto de ApS.

- Entrevista estructurada final con el profesorado responsable.

- Rúbrica de autoevaluación para el profesorado.

- Entrevista estructurada con los responsables de la entidad receptora del servicio.

- Grupo de discusión con los profesores responsables de los proyectos.

- Evaluación de seguimiento o 
impacto, a partir de indicadores como la continuidad del proyecto, la demanda de formación por parte del profesorado, la evaluación del docente implicado, etc.

\section{Conclusiones}

En este trabajo presentamos una investigación de alcance experimental con la que pretendemos ayudar a sentar las bases para una institucionalización sostenible del aprendizaje servicio en las universidades españolas. En tal sentido, es obligado reconocer el escaso número de investigaciones sólidas existentes en nuestro país sobre aprendizaje servicio y universidad, cuestión que incluso se ha podido evidenciar en congresos y conferencias recientes sobre el tema. Por ello, la envergadura del proyecto, en el que se estudian 20 proyectos de aprendizaje servicio, y en el que se emplean diversos instrumentos cuantitativos y cualitativos para comparar los resultados en alumnos participantes en ApS y no participantes, antes y después del servicio comunitario, supone un avance en la investigación sobre esta práctica educativa en el contexto español.

Por lo tanto, lo que buscamos con esta investigación es mostrar el nivel de implementación del ApS en el sistema universitario de nuestro país. Siendo así, lo que precisamos es conocer aquellos asuntos de la institución que se relacionan con la exploración, la implementación, la expansión y la sostenibilidad del aprendizaje servicio en términos programáticos (Lambright y Alden, 2012; Santos Rego y Lorenzo,
2007). En mucho casos, afirma una reconocida experta del ámbito latinoamericano (Tapia, 2010), el aprendizaje servicio se desarrolla a través de procesos de transición, no siempre intencionados, que parten de otro tipo de actividades pero que no llegan a definirse como tal.

Este estudio supone un avance significativo, ya que la investigación en el campo se ha caracterizado por la ausencia de estudios que empleen diseños experimentales, por utilizar muestras pequeñas y porque buena parte de los estudios se basan en percepciones de alumnos y profesores (Furco, 2007). En pocas palabras, es claro que uno de los elementos fundamentales vinculados a la pertinencia del aprendizaje servicio como marco de innovación y desarrollo de los procesos de aprendizaje en las universidades españolas vendrá dado por la demostración empírica de que la introducción de este tipo de programas en la educación superior optimiza el rendimiento académico y mejora las competencias sociales y destrezas cívicas de los estudiantes, sobre las que existe bastante consenso en tanto que aspectos sustantivos en las dinámicas de inserción laboral.

En definitiva, lo que defendemos es que las tentativas institucionalizadoras de la metodología ApS en el sistema universitario español deberían apoyarse en cuatro pilares básicos:

1. Los hallazgos de la investigación social, sobre todo en la escena internacional, que asocian el ApS a la mejora del rendimiento académico y la 
elevación del capital social de los estudiantes.

2. El marco legal e institucional que ampara en ApS en el sistema universitario español y, naturalmente, en el EEES.

3. Cada vez son más los profesores que trabajan con esta metodología, muchas veces de forma aislada y a modo de iniciativa personal, pero sin un respaldo institucional y pedagógico que permita afirmar la sostenibilidad de tales iniciativas.

4. Los beneficios que se derivan de la institucionalización de cualquier práctica frente a aquellas que no cuentan con ese reconocimiento (Furco, 2007).

\section{Referencias bibliográficas}

Astin, A. W., Vogelgesang, L. J., Ikeda, E.K., y Yee, J. A. (2000). How servicelearning affects students. Los Angeles: Higher Education Research Institute. Recuperado de https://heri.ucla.edu/PDFs/HSLAS/HSL AS.PDF

Bringle, R. G., y Hatcher, J. A. (2000). Institutionalization of service learning in higher education. The Journal of Higher Education, 71(3), 273-290. doi: $10.2307 / 2649291$

Buenestado, M., Álvarez, J. L., y González, H. (2016). Análisis de las actividades de formación docente de las universidades españolas relacionadas con la metodología aprendizaje-servicio. En M. A. Santos Rego, A. Sotelino Losada, y M. Lorenzo Moledo (Eds.), Aprendizaje-servicio e innovación en la universidad. Actas del VII Congreso Nacional y II Internacional de Aprendizaje-Servicio Universitario (pp. 125-134). Santiago de Compostela: Universidad de Santiago de Compostela.

Furco, A. (2003). Issues of definition and program diversity in the study of service-learning. En S. H. Billig, y A. S. Waterman (Eds.), Studying servicelearning. Innovations in education research methodology (pp. 13-33). Mahwah, NJ: Lawrence Erlbraum Associates.

Furco, A. (2005). Impacto de los proyectos de aprendizaje servicio. Aprendizaje y servicio solidario en la Educación Superior y en los sistemas educativos latinoamericanos. Actas del 70 Seminario Internacional Aprendizaje y Servicio Solidario, Buenos Aires (Argentina), 19-26. Recuperado de http://www.clayss.org.ar/seminario/ant eriores/actas/2005_Actas7.pdf

Furco, A. (2007). Institutionalising service-learning in higher education. En L. McIlrath, e I. M. Labhrainn (Eds.), Higher education and civic engagement: international perspectives. (pp. 65-82). Aldershot: Ashgate.

Furco, A. (2011). Rúbrica de autoevaluación para la Institucionalización del AprendizajeServicio en la Educación Superior. Recuperado de http://educaciocp89.webjoomla.es/wpcontent/uploads/04-Furco-2Castellano.pdf 
Gallini, S. M., y Moely, B. E. (2003). Service-learning and engagement, academic challenge, and retention. Michigan Journal of Community Service Learning, 10(1), 5-14. Recuperado de http://hdl.handle.net/2027/spo.323952 1.0010.101

Howard, J. (2003). Service-learning research: Foundational issues. En S. H. Billig, y A. S. Waterman (Eds.), Studying service-learning. Innovations in education research methodology ( $\mathrm{pp}$. 1-12). Mahwah, NJ: Lawrence Erlbraum Associates.

Lambright, K., y Alden, A. F. (2012). Voices from the trenches: faculty perspectives on support for sustaining service learning. Journal of Higher Education Outreach and Engagement, 16(2), 9-45. Recuperado de http://openjournals.libs.uga.edu/index. php/jheoe/article/view/788/554

Martínez, M. (2008). Aprendizaje servicio y construcción de ciudadanía activa en la universidad: la dimensión social y cívica de los aprendizajes académicos. En M. Martínez Martín (Ed.), Aprendizaje servicio y responsabilidad social de las universidades (pp. 27-56). Barcelona: Octaedro.

McIlrath, L., Lyons, A., y Munck, R. (Eds.) (2012). Higher education and civic engagement. Comparative perspectives. New York, NY: Palgrave Macmillan.

Pérez, C., Vázquez, V., y López, I. (2016). El Aprendizaje-Servicio en las Guías Docentes de las titulaciones universitarias. En M. A. Santos Rego, A. Sotelino Losada, y M. Lorenzo Moledo (Eds.), Aprendizaje-servicio e innovación en la universidad. Actas del VII Congreso Nacional y II Internacional de Aprendizaje-Servicio Universitario (pp. 653-666). Santiago de Compostela: Universidad de Santiago de Compostela.

Puig, J. M. (Coord.) (2009). Aprendizaje Servicio (ApS). Educación y compromiso cívico. Barcelona: Graó.

Puig, J. M., Palos, J., Martín, X., Rubio, L., y Escofet, A. (2016). Aprendizaje servicio e innovación en la Universidad. En M. A. Santos Rego (Ed.), Sociedad del conocimiento. Aprendizaje e innovación en la Universidad (pp. 155178). Madrid: Biblioteca Nueva.

Santos Rego, M. A., Jover, G., Naval, C., Álvarez, J. L., Vázquez, V., y Sotelino, A. (en prensa). Diseño y validación de un cuestionario sobre práctica docente y actitud del profesorado universitario hacia la innovación (CUPAIN). Educación XX1, 20(2). doi: 10.5944/educXX1

Santos Rego, M. A., y Lorenzo, M. (2007). Universidade e construcción da sociedade civil. Vigo: Xerais.

Santos Rego, M. A., Sotelino, A., y Lorenzo, M. (2015). Aprendizajeservicio y misión cívica de la universidad. Una propuesta de desarrollo. Barcelona: Octaedro.

Stater, K. J., y Fotheringham, E. (2009). Mechanisms for institutionalizing service-learning and community partner 
outcomes. Journal of Higher Education Outreach and Engagement, 13(2), 729. Recuperado de http://openjournals.libs.uga.edu/index. php/jheoe/article/view/84/72

Tapia, M. N. (2004). Aprendizaje y servicio solidario. Madrid: Editorial CCS.

Tapia, M. N. (2010). La propuesta pedagógica del aprendizaje-servicio: una perspectiva latinoamericana.

Tzhoecoen. Revista Científica, 3(5), 2343. Recuperado de http://www.clayss.org.ar/04_publicacio nes/TZHOECOEN-5.pdf

Ugarte, C., y Naval, C. (2010). El service-learning, medio adecuado para promover la cooperación social. El caso concreto del programa Create your own charity. En C. Naval, S. Lara, C. Ugarte, y Ch. Sádaba (Eds.), Educar para la comunicación y la cooperación social (pp. 263-281). Pamplona: Consejo Audiovisual de Navarra.

Nota

Este trabajo se deriva del Proyecto de $\mathrm{I}+\mathrm{D}+\mathrm{i}$ "Aprendizaje-Servicio e Innovación en la Universidad. Un programa para la mejora del rendimiento académico y el capital social de los estudiantes", financiado por el Ministerio de Economía y Competitividad y fondos FEDER como proyecto de $\mathrm{I}+\mathrm{D}+\mathrm{i}$ Retos Investigación del Programa Estatal de I+D+i Orientada a los Retos de la Sociedad (EDU2013-41687-R). 